\title{
GESTIÓN GERENCIAL DEL DIRECTOR Y LA RESPONSABILIDAD SOCIAL EN LAS ORGANIZACIONES EDUCATIVAS.
}

\author{
Amílcar R. ${ }^{1}$ La Cruz Zambrano, Violeta Pérez Ruz²
}

\section{Resumen}

El propósito de la presente investigación fue generar lineamientos prácticos para la Gestión Gerencial del director y la Responsabilidad Social en las organizaciones educativas del Estado Zulia-Venezuela. Se apoyó teóricamente en autores como Koontz y Weihrich (2006), Chiavenato (2006), García (2003) entre otros. El paradigma de investigación fue positivista, metodología cuantitativa; el tipo descriptiva, proyectiva. El diseño investigación de campo, no experimental. La población se conformó por un censo poblacional 12 directivos y en cuanto a los docentes, se tomo una muestra a través de la formula de Sierra Bravo; quedando conformada por 127 sujetos. Se elaboró un cuestionario, lo cual fue tipo encuesta, con constituido por 18 itemes y 04 opciones o categorías de respuestas, el cual fue validado por cinco expertos y la confiabilidad se obtuvo a través del coeficiente Alfa de Cronbach, obteniendo un puntaje de $r=0,90$. Los resultados se analizaron utilizando una estadística descriptiva los cuales indicaron inexistencia de lineamientos para el fortalecimiento de las Gestión Gerencial del director y la Responsabilidad Social desde las organizaciones educativa objeto de estudios. Se recomendó la puesta en práctica de los lineamientos.

Palabras clave: Gestión gerencial, Responsabilidad social, Organizaciones Educativas.

${ }^{1}$ Profesor en Educación Técnica. Especialista en Gerencia Educativa. Doctor en innovaciones Educativa. Acreditado por el Ministerio de Ciencia y Tecnología como Investigador A. Docente Contratado categoría Titular de URBE, UPEL, Tutor Docente a Distancia modalidad Virtual Universidad del Magdalena. Correo Electrónico: amilcalacruz12@gmail.com

${ }^{2}$ Licenciada en educación. Especialista en Gerencia Educativa. Especialista en Metodología de Investigación. Doctora en Innovaciones Educativa. Acreditada por el Ministerio de Ciencia y Tecnología como Investigadora A. Docente Contratado categoría Titular de modalidad Presencia y a distancia (Virtual) URBE, UPEL, Correo Electrónicovperezruz@hotmail.com 


\section{MANAGING DIRECTOR MANAGEMENT AND SOCIAL RESPONSIBILITY FROM OF EDUCATIONAL ORGANIZATIONS.}

\section{Abstract}

The purpose of this research was to generate practical guidelines for strengthening Managerial Management and Social Responsibility Director from of educational organizations Zulia State-Venezuela. It is theoretically supported and Weihrich authors like Koontz y Weihrich (2006), Chiavenato (2006), García (2003) among others. The research paradigm was positivist, quantitative methodology, the descriptive, projective. The field research design, not experimental. The population was comprised of a population census as 12 officers and teachers, will take a sample by the formula of Sierra Bravo, being conformed by 127sujetos. A questionnaire was elaborated, which was kind survey, with consists of 42 itemes and 04 options or categories of responses, which was validated by five experts and the reliability was obtained by Cronbach's alpha coefficient, obtaining a score of $r=0.90$. The results were analyzed using descriptive statistics which indicated lack of guidelines for strengthening the director Managerial Management and Social Responsibility from of educational organizations under study. It recommended the implementation of the uidelines.

Keywords: Administrative management, social responsibility, Educational Organizations.

\section{Introducción}

Los cambios que se generan en la actualidad, demanda la presencia de un líder holístico, una persona cuyas competencias en cuanto a conocimiento, habilidades, destrezas y actitudes conduzcan los procesos gerenciales por el camino correcto al dirigir al personal de manera que se cumplan con todas las exigencias, teniendo relevancia el papel del director que como lo expone Joel J. Lerner y H.A. Baker citado por Galindo y Martínez (1998:147), consiste en dirigir las operaciones mediante la cooperación del esfuerzo de los subordinados, para obtener altos niveles de productividad mediante la motivación y la supervisión.

Por otra parte Requeijo (2013) manifiesta que el Director es el representante del Ministerio de Educación ante la comunidad, y al mismo tiempo responsable de establecer las buenas relaciones entre sus miembros y con los demás sectores involucrados en el proceso educativos a fin de incorporarlos a todos en el desarrollo del mismo. Es decir, una persona que observa desde arriba a un grupo de trabajadores o un equipo y ayuda a sus miembros a desarrollar un trabajo que cree valor para sus clientes; garantizando la calidad de los procesos educativos.

Por lo tanto, la gestión gerencial se refiere al proceso emprendido por el director para coordinar las actividades laborales de estas personas con la finalidad de lograr resultados de alta calidad que cualquiera trabajando solo no podría lograr. Por tal razón, la gestión o acción a desarrollar por el director en las organizaciones educativas es procurar en todo momento mantener condiciones de eficacia y eficiencia que lo conduzcan al logro de los objetivos educacionales, el cual es la formación integral del ser humano, que a su vez permitirá que el hombre logre interiorizar 
el cumulo de valores, forjar su personalidad, no solo dentro del ámbito productivo, sino también para la convivencia y dentro de ella el compromiso social. Se plantea al respecto:

La educación es una de las dimensiones fundamentales, su finalidad es la de formar al individuo para que asuma las transformaciones que la sociedad demanda, capacitándolo en la responsabilidad social, productiva, la participación ciudadana y la consolidación de valores democráticos necesarios para avanzar en los procesos de cambio (Finol, 2001:3).

Aludiendo a la afirmación anterior, es necesario hablar entonces que, la responsabilidad social, definida por Chiavenato (2006:204), como el grado de obligaciones que una organización asume por medio de acciones que proyectan y brinden bienestar a la sociedad a medida en que busca alcanzar sus propios intereses. En general representa la obligación de la organización de adoptar políticas y asumir decisiones y acciones que beneficien a la sociedad; por lo tanto el término de responsabilidad social es también aplicable a las organizaciones educativas, ya que pretenden que ésta contribuya activa y voluntariamente en el mejoramiento social, económico y ambiental.

En este orden de ideas, los investigadores plantean que las instituciones educativas, deben contribuir al mejoramiento social a través de la correcta formación de los estudiantes en cuanto a conocimientos, habilidades y destrezas, fomentando en ellos valores éticos, además de formar e integrar a los padres y representantes en el proceso educativo de sus hijos. Igualmente planificar y organizar acciones para mejorar la calidad de vida de la población, así mismo promover y desarrollar en la población la conservación del entorno donde vive.
En Venezuela el Ministerio del Poder Popular para la Educación, cuya gestión educativa está orientada hacia el fortalecimiento de un nuevo paradigma educativo el cual contempla la gestión gerencial ha de ser ejecutada por los directivos, como actores principales para el fortalecimiento de la responsabilidad social, tal parece que algunos directores de las organizaciones educativas poseen escasos conocimientos en gerencia, utilizando un estilo gerencial orientado solo a los procesos administrativos, esta situación podría generar un desequilibrio en la eficiencia y eficacia de toda la funcionalidad de la organización educativa, asimismo pareciera que la gestión gerencial del director presentan serias debilidades, por cuanto que este personal muestra un comportamiento ético que dista mucho del deber ser.

De igual manera, se presume que se estuviera obviando el compromiso de la responsabilidad social, derivado en algunos casos porque algunos miembros del personal directivo de estas organizaciones educativas, no asumen acciones encaminadas al bienestar de la comunidad donde se encuentra inmersa, en consecuencia dirigen la institución en forma unilateral, por lo tanto las decisiones no son consultadas y las prioridades no siempre concuerdan con la realidad institucional y por ende social.

Por otro lado, el personal directivo y docente, posiblemente presentan una frágil función como líderes sociales, lo cual impide la integración de la comunidad a los intereses educacionales, observándose al respecto, algunas conductas que hacen suponer la falta de iniciativas por parte del personal a un acercamiento a las fuerzas vivas de la comunidad. Así mismo, le dan poca importancia a la elaboración, ejecución y evaluación del Proyecto Educativo Comunitario, lo cual limita el cumplimiento de estos ámbitos, puesto que no se crean redes institucionales entre escuela y comunidad para dar solución a los problemas que aquejan a las organizaciones educativas y su entorno. 
En este orden de ideas, probablemente estos centros educativos no responden a los requerimientos de los elementos de la responsabilidad social educativa como lo son: compromiso hacia la sociedad venezolana debido a que le agrega poco valor, presentan esquemas tradicionales dificultándosele la adaptabilidad al cambio del ambiente interno y externo además conciben la responsabilidad social como una obligación más no como una acción voluntaria. En relación a lo planteado se hace pertinente formular y dar respuesta a la siguiente interrogante: ¿Cuáles serían los lineamientos prácticos para la Gestión Gerencial del director y la Responsabilidad Social en las organizaciones educativas?

Por tal motivo, se plantea la necesidad de establecer un ambiente educativo contextualizado donde el conductor y mediador de los procesos académicos, administrativo y técnicos tengan un amplio conocimiento teórico, metodológico y operativo a fin de garantizar el éxito de la calidad del proceso educativo involucrando para ello, a docentes y a la comunidad educativa en general.

Finalmente, la presente investigación se plantea como Objetivo Principal generar lineamientos prácticos para la Gestión Gerencial del director y la Responsabilidad Social en las organizaciones educativas. Asimismo, para el desarrollo del objetivo principal es necesario responder a los Objetivos Específicos como Describir los procesos de la gestión gerencial aplicados por el director para el desempeño efectivo en las organizaciones educativas; así como explicar los elementos de la responsabilidad social que han de estar presentes en las organizaciones educativas y Proponer líneas de acción en la gerencia educativa para el impulso de la Responsabilidad Social en las organizaciones educativas.

Para el desarrollo de los fundamentos teóricos de esta investigación, se consideró conveniente estudiar una serie de aspectos relevantes con las variables de estudio, con el objeto de cons- truir un sustento teórico lo cual es indispensable para una mejor comprensión y entendimiento de los mismos.

\section{Gestión Gerencial del Director}

El director es la persona que planifica, organiza y controla una organización con el fin de conseguir en el campo educativo el beneficio social, la formación de recursos humanos. Según Fernández (2001:23), cuando se administra una institución los directores deben poner en práctica sus habilidades gerenciales, cuya tarea es la creación de un ambiente donde los individuos trabajen en equipo y puedan desempeñarse en forma eficaz y eficiente para la obtención de fines comunes. Afirma también el autor que es también importante una buena preparación en procesos gerenciales para garantizar una gestión exitosa. En la gestión gerencial llevada a cabo por los directores, es importante considerar los procesos administrativos, las acciones y estrategias desarrolladas en las instituciones educativas

Para el ejercicio del rol, el director debe tener habilidades que le permitan asumir el cumplimiento de los procesos inherentes a su cargo. En múltiples oportunidades se le concede mayor importancia, con el fin de conseguir la unidad entre las personas y la organización. Según Chiavenato (2006:127), «los procesos gerenciales de un gerente están dirigidos a procurar integrar, que los distintos elementos de la organización alcancen un estado de equilibrio».

De allí, que el desempeño gerencial se refiere al proceso emprendido para coordinar las actividades laborales de estas personas con la finalidad de lograr resultados de alta calidad, por tal razón, las acciones desarrolladas por el director en las instituciones deben procurar en todo momento mantener condiciones de eficacia y eficiencia que le conduzcan al logro de objetivos institucionales, esta acción se define como desempeño, la cual es para Chiavenato (2006:178), 
la eficacia del personal que labora dentro de las organizaciones es necesaria, porque con ella la organización debe funcionar y el individuo debe lograr satisfacción.

Para Ruetter y Conde (2004:28), el papel del director debe «vitalizar, renovar y activar permanentemente los recursos humanos y técnicos a través de la motivación y el desarrollo individual que orienten la acción y resolución de problemas en concordancia con los objetivos de la institución». Esto significa concebir a la organización como un sistema socio-técnico, compuesto a la vez por un subsistema social en el que está presente la dimensión humana. De forma tal para que el director pueda realizar la labor de asesorar, orientar y administrar los procesos que se desarrollan en los planteles, es necesario que posea una adecuada formación gerencial.

Dentro de las exigencias que se le solicita al director en su redimensión laboral en aras de que en su trabajo sea conductor de las políticas educativas, se plantee el manejo de procesos gerenciales que son cotidianos en la dinámica dentro de una institución escolar. A efecto de desarrollar una gestión de calidad eficiente y eficaz como lo plantea Chiavenato (2006:184), la comunicación como un proceso, está más orientado como un requisito o habilidad que debe desarrollar el director; esta constituye en la práctica el inicio de todos los procesos gerenciales.

El manejo adecuado de la comunicación organizacional le puede permitir al director logros en buen discurso resultando convincente y alentador, un mensaje puede ser altamente motivador. A partir de aquí puede organizar de manera más efectiva al personal al establecer una comunicación cara a cara resulta para los miembros de la organización, más comprometedora porque la transmisión se está dando de manera directa. Es importante por ende, entender la comunicación como una herramienta que permite el conocimiento entre las per- sonas, mejora y fortalece las relaciones entre directivos, docentes y estudiantes, sensibiliza hacia las diferencias individuales de personas y grupos, entre otros aspectos.

Es importante destacar que la dinámica de una organización tiene inmerso en ella una serie de acciones que cumplir bajo ciertos parámetros que el director con una misión y visión clara definida prevé planes emergentes que pueda poner en práctica en momentos determinados para lograr solventar situaciones inadecuadas para el desarrollo de la institución que dirige. Por lo tanto, Koontz y Weiluich. (2006:112), expresan que la toma de decisiones es «la selección de un curso de acción entre varias alternativas y constituye, por tanto, la esencia de las planificaciones o proyectos organizacionales».

Lo expuesto quiere decir que no se puede determinar un plan de acción sino se ha tomado una decisión acertada en compromiso de parte de todas las personas involucradas en un problema de la organización, una revisión de recursos o una dirección acertada y esta situación por lo general aparece en todos los gerentes directivos, pues son ellos quienes deciden que hacer, quien lo hará, cuando, dónde y cómo se hará en la organizaciones educativas.

Por otra parte, para entender el funcionamiento de las organizaciones es importante el estudio de la realidad sobre la manera como el personal desarrolla sus labores. En este sentido es prudente entender la capacidad de colaborar y cooperar con los demás. Toda organización es fundamentalmente un equipo por sus miembros. Al respecto, Gento (2000:150), considera que el trabajo en equipo puede entenderse como: «el resultado de la actividad de un conjunto de personas que actúan con un método, tiene un objetivo en común y la responsabilidad sobre el resultado».

De allí que el trabajo en equipo es otro proceso donde el director puede delegar y a su vez 
reconocer las potencialidades del personal al asignarle responsabilidades en quipo. La gestión gerencial de un director no puede estar exenta de un estilo de liderazgo definido, que marca pautas de comportamiento y conducción dentro de la institución. Al respecto, Mosley, Megginson y Pietri (2005:24), definen el liderazgo como «el proceso de influir sobre las actividades individuales y de grupo en la fijación y consecución de metas».

Este trabajo en equipo parte del compromiso de cada uno de los miembros del grupo para contribuir con su esfuerzo y en la medida que les corresponda a impulsar la consecución de los objetivos comunes, pero para que esta contribución sea equitativa y con incidencia eficaz sobre los resultados, conviene que se lleve a cabo de acuerdo con una distribución previa de las responsabilidades a cubrir; de este modo la contribución solidaria tendrá un efecto multiplicador sobre el esfuerzo de todos y cada uno de los miembros.

\section{Elementos de Responsabilidad Social}

La responsabilidad social es la contribución activa y voluntaria de las empresas al mejoramiento social, económico y ambiental. Según García (2003:54), es «la obligación gerencial de tomar las medidas que protejan y mejoren el bienestar de la sociedad como un todo y también los intereses organizacionales». Se deduce que este término hace referencia al conjunto de obligaciones y compromisos legales y éticos del personal que labora en la organización tanto nacionales, estadales y privadas que se derivan de los impactos de las actividades de la escuela, las cuales se producen en los ámbitos social, laboral, medioambiental y de los derechos humanos

Dentro de este marco de ideas, las organizaciones educativas son cada vez más conscientes de la necesidad de incorporar las preocupaciones sociales, laborales, económicas, ambienta- les y los derechos humanos como parte de su estrategia educacional. Por lo tanto, el director puede utilizar diferentes modalidades para inculcar en las labores cotidianas, la responsabilidad social en sus estudiantes, docentes y demás personal; cumpliendo con las expectativas deseadas por la sociedad. En lo disciplinario respetando las reglas de convivencia propia de la institución donde pertenece, fomentando la creatividad y generando el espacio para el desarrollo social, científico, político y económico en pro de la sociedad en la que se vive.

Por su parte, la comunidad tendrá acceso al capital intelectual desarrollado dentro de las instituciones educativas. Se abre sí las puertas para las dos partes para examinar los puntos de vistas propios y para recibir información que puede ser útil, estas experiencias llevan a la formación de ciudadanos que empiecen a experimentar desde su etapa académica, los problemas reales del país, y que consiguientemente, comiencen a valorar y a participar activamente en lo político y lo social. En este orden de ideas y siguiendo a García (2003:155), pueden identificarse elementos importantes que comparten las diferentes definiciones de responsabilidad social a saber: el compromiso, la acción voluntaria y la adaptabilidad al cambio.

Dentro de este marco de ideas, García (2003:74), señala que el compromiso con la sociedad se refiere a las diferentes acciones tomadas por la empresa para maximizar el impacto de las contribuciones, «en dinero, tiempo, producto, servicios, influencias, administración del conocimiento y otros recursos que dirige hacia las comunidad en las cuales opera». Ante los planteamientos señalados con anterioridad, el compromiso de las organizaciones educativas como forma de responsabilidad social, tiene que ver con la forma de administrar y tomar decisiones para alcanzar o superar las expectativas éticas, legales y sociales que la sociedad tiene de la misma. 
Seguidamente, la capacidad para la adaptabilidad al cambio es lo que caracteriza a una organización exitosa. Para Robbins, S (2004:630), define cambio como el conjunto de variaciones de orden estructural que sufren las organizaciones, que se traducen en un nuevo comportamiento organizacional. Por su parte Collerette y Delisle (2001) cambio se refiere a una modificación observable que ocurrió en el sistema social. Es conveniente resaltar que deberá estar igualmente capacitado para asesorar, promover y mantener programas, acciones dirigidas al funcionamiento de las instituciones, estableciendo un clima de confianza del grupo que lo acompaña en su gestión.

Por otra parte, otro de los elementos esenciales de la responsabilidad social, es la acción voluntaria, el cual representa el carácter exclusivamente voluntario de la responsabilidad social no regulada por ley; según la definición de responsabilidad social citada por el Libro Verde de la Unión Europea (2005:6), se encuentra que es un concepto por el cual las empresas «deciden contribuir voluntariamente a mejorar la sociedad y a preservar el medio ambiente». En este sentido, el voluntariado en la acción voluntaria, es el resultado de libre elección por parte de cada miembro del plantel, es una opción ética, personal, gratuita que no espera retribución o recompensa.

\section{Métodos}

El enfoque metodológico en este sentido, de acuerdo con Pérez (2000) se inserta en el para- digma positivista, porque se obtuvo una medida cuantitativa de las variables, dimensiones, indicadores e ítems. En tal sentido, la investigación es abordada, según Hernández y otros (2006) bajo las características de los estudios descriptivos; lo cual buscan especificar las propiedades y características o cualquier otro fenómeno que se someta a un análisis.

Según el nivel de profundidad se sustenta en el tipo investigación proyectiva (Hurtado, 2000). Plantea que consiste en la elaboración de una propuesta o de un modelo, para solucionar problemas o necesidades de tipo práctico, ya sea de un grupo social, institución, un área en particular del conocimiento, partiendo de un diagnóstico preciso de las necesidades del momento y según Chávez (2006: 133) su propósito se considero aplicada; ya que tiene como fin principal resolver un problema en un periodo de tiempo corto.

Así mismo, el estudio se enmarco dentro de un diseño no experimental, según Kelinger (2002) citado por Hernández (2006) manifiesta que el investigador no posee control directo de la variable; y es de campo dado que la misma se desenvolvió en el sitio donde se llevaron a cabo los eventos a estudiar. En este sentido la población objeto de estudio está constituido, por el personal directivo y docentes de las Organizaciones Educativas del Municipio Escolar San Francisco 2 del Estado Zulia-Venezuela.

Tabla $N^{\circ}$ 1. Distribución de la Población

\begin{tabular}{lcc} 
Organizaciones Educativas & Directivos & Docentes \\
\hline Carmela Quintero & 2 & 34 \\
Dr. Jesús Muñoz Tebar & 3 & 37 \\
Gran Mariscal de Ayacucho & 3 & 61 \\
Felipe Neri Sandrea & 2 & 48 \\
Emma Elisa Finol & 2 & 45 \\
TOTAL & $\mathbf{1 2}$ & $\mathbf{2 2 5}$ \\
\hline
\end{tabular}

Fuente: Investigación de los autores 
La población de directivos se considera finita. Según Pérez, L. (1997) es la que contiene un numero finito de unidades, no es infinitamente grande. Así mismo, se caracteriza por ser censo poblacional, apoyándonos en las afirmaciones de Hernández y otros, (2006: 238), «... la selección de elementos depende del criterio del investigador». Se considero pertinente tomar la población total de los directivos por considerarse finita, ya que en ello se son los primeros en identificarse con el problema, así mismo. «es el recuento de todos los elementos de la población», para la población de los docentes (225), a la cual se realizó el muestreo. Para Chávez (2007:162), la muestra es una «porción representativa de la población que permite generalizar sobre esta, los resultados de una investigación». Según Hernández y otros (2006:206), por lo tanto, Para calcular la muestra se asume la fórmula de Sierra Bravo (2000:183):

$$
n=\frac{4 N p q}{E^{2}(N-1)+4 p q}
$$

En esta investigación el muestreo es probabilístico y estratificado para calcular se consideró la formula de Schiffer quedando conformado por 127 docentes.

\section{Técnicas e instrumentos de recolección de datos}

En este estudio de campo, la encuesta se presentó como la técnica más adecuada para recolectar la información. Según Hurtado (2000) es basada en la interacción personal y se utiliza cuando la información requerida por el investigador es conocida por otras personas o cuando lo que se investiga forma parte de la experiencia de esas personas. En cuanto a los instrumentos empleados fue el cuestionario afirma (Sierra, 2004: 369), que no es otra cosa que un conjunto de preguntas estructuradas, preparadas cuidadosamente sobre los hechos, aspectos que interesan en una investigación Los instrumento quedo estructurado para cuarenta y dos (18) ítems.

\section{Resultado de la investigación}

Tabla $N^{\circ}$ 2. Variable: Gestión Gerencial y Responsabilidad Social

Dimensión: Procesos en Gestión Gerencial y Elementos Responsabilidad Social

\begin{tabular}{|c|c|c|c|c|c|c|c|c|}
\hline \multirow{2}{*}{$\begin{array}{l}\text { Alternativa } \\
\text { Población }\end{array}$} & \multicolumn{2}{|c|}{ Siempre } & \multicolumn{2}{|c|}{$\begin{array}{c}\text { Casi } \\
\text { siempre }\end{array}$} & \multicolumn{2}{|c|}{ Casi nunca } & \multicolumn{2}{|c|}{ Nunca } \\
\hline & Direc & Doc & Direc & Doc & Direc & Doc & Direc & Doc \\
\hline \% Dimensiones & $\%$ & $\%$ & $\%$ & $\%$ & $\%$ & $\%$ & $\%$ & $\%$ \\
\hline $\begin{array}{l}\text { Procesos en la Gestión } \\
\text { Gerencial }\end{array}$ & 49.89 & 5.95 & 38.80 & 37.70 & 11.11 & 53.10 & 0 & 3.10 \\
\hline $\begin{array}{l}\text { Elementos de la } \\
\text { Responsabilidad Social }\end{array}$ & 38.80 & 6.73 & 51.77 & 39.88 & 8.25 & 49.90 & 0 & 2.4 \\
\hline Promedio & 44.34 & 6.34 & 45.28 & 38.79 & 9.68 & 51.5 & 0 & 2.75 \\
\hline Porcentaje & \multicolumn{2}{|c|}{25,34} & \multicolumn{2}{|c|}{42.03} & \multicolumn{2}{|c|}{30.59} & \multicolumn{2}{|c|}{2.75} \\
\hline
\end{tabular}

Fuente: Elaboración propia. 
Nótese en la tabla $\mathrm{N}^{\circ} 2$, en los resultados para definir las dimensión Procesos Gerenciales se observa que la mayor frecuencia se presentó en un $53.10 \%$, por parte del personal docente en la alternativa casi nunca, observándose una debilidad en la dimensión Procesos en la Gestión Gerencial evidenciado un vacío conceptual en la praxis gerencial de acuerdo a la evaluación realizada por los docentes, mientras que 49.89 $\%$ los directivos consideraron la alternativa Siempre cumplen con dichos procesos.

En relación a la variable Elementos de la Responsabilidad Social, se observó que los docentes se ubicaron en una frecuencia de $49.90 \%$ manifestando que casi nunca se vinculan a la dimensión; situación que refleja carencia en los gerentes educativos en cuanto a la integración y proyección a la comunidad, mientras que por parte del personal directivo presentan una frecuencia de 51.77 \% ubicándose en la alternativa siempre cumplen con dichos elementos.

\section{Discusión}

Por consiguiente, los resultados están en contraposición con los postulados teóricos de Fernández (2001:23), cuando se administra una institución los directores deben poner en práctica sus habilidades gerenciales, cuya tarea es la creación de un ambiente donde los individuos trabajen en equipo y puedan desempeñarse en forma eficaz y eficiente para la obtención de fines comunes. Afirma también el autor que es también importante una buena preparación en procesos gerenciales para garantizar una gestión exitosa.

En la gestión gerencial llevada a cabo por los directores, es importante considerar las acciones y estrategias desarrolladas en las organizaciones educativas. De allí que el desempeño gerencial se refiere al proceso emprendido para coordinar las actividades laborales de estas personas con la finalidad de lograr resultados de alta calidad, por tal razón, las acciones desarrolladas por el supervisor en las instituciones deben procurar en todo momento mantener condiciones de eficacia y eficiencia que le conduzcan al logro de objetivos institucionales, esta acción se define como desempeño.

De acuerdo, a Chiavenato (2006:178), la eficacia del personal que labora dentro de las organizaciones es necesaria, porque con ella la organización debe funcionar, el mismo autor señala que «los procesos gerenciales de un gerente están dirigidos a procurar integrar, que los distintos elementos de la organización alcancen un estado de equilibrio».

Por tal motivo, los procesos gerenciales constituyen elementos importantes que permiten al supervisor darle fluidez a las actividades institucionales. De igual manera difieren de los planteamientos de Mosley, Magginson y Pietri (2005:9), consideran que todo director cumple funciones administrativas «las cuales influyen una amplia clasificación de las actividades que desempeña todo gerente», por tanto en este orden, debe cumplir las funciones de manera efectiva.

Por otra parte, los resultados están en contraposición con los postulados teóricos de García (2003:54), al referir que responsabilidad social es «la obligación gerencial de tomar las medidas que protejan y mejoren el bienestar de la sociedad como un todo y también los intereses organizacionales». Se deduce que este término hace referencia al conjunto de obligaciones y compromisos legales y éticos del personal que labora en la organización tanto nacionales, estadales y privadas que se derivan de los impactos de las actividades de la escuela, las cuales se producen en los ámbitos social, laboral, medioambiental y de los derechos humanos.

Por tal motivo, las organizaciones educativas son cada vez más conscientes de la necesidad de incorporar las inquietudes sociales, 
laborales, económicas, ambientales y los derechos humanos como parte de su estrategia educacional. García (2003:57), manifiesta, pueden identificarse elementos importantes que comparten las diferentes definiciones de responsabilidad social a saber: el compromiso, la acción voluntaria y la adaptabilidad al cambio. Por lo tanto, la gestión gerencial debe estar orientada a la aplicación de estrategia y acciones que faciliten el trabajo en equipo de todos los miembros de la comunidad educativa para solucionar los problemas institucionales y sociales de manera consensuada y en el orden de prioridades

\section{Líneas de acción en la gerencia educativa para el impulso de la Responsabilidad Social en las organizaciones educativas}

Los presentes lineamientos están dirigidos a los directores de las organizaciones educativas y deberán aplicarse en el desarrollo de programas, planes y proyectos para generar cambios positivos en el fortalecimiento de la gestión gerencial lo cual está dado por:

- Planificar reuniones para realizar un diagnóstico de las necesidades y fortalezas de la institución y la comunidad.

- Organizar equipos multidisciplinario (psicólogos, sociólogo, docentes, entre otros) que planifiquen actividades recreativas, culturales, ambientales, educativas dirigidas a resolver las debilidades presentes.

- Elaborar medios informativos para toda la comunidad con la ayuda de la tecnología de información y comunicación y el proyecto Canaima presentes en las instituciones.

- Se debe mantener constante la promoción de los principios axiológicos como son la dignidad, el respeto, la justicia y la solidaridad, la tolerancia y la autoestima fortaleciéndolos y vivenciándolos permanentemente por medio de todas las áreas del conocimiento.

- Garantizar la participación de las comunidades en los proyectos de aula y proyectos plantel.
- Trabajar en conjunto con los consejos comunales y fuerzas vivas comunitarias para establecer lazos de integración que permitan resolver los problemas que afectan a la comunidad y organización educativa.

Por tal motivo, el director ha de promover los correctos valores y así fortalecer la responsabilidad social la cual debe estar presente en toda la organización educativa.

\section{Conclusiones}

Se evidencio que la mayoría de directivos presentan dificultades en el cumplimiento en los procesos de la gestión gerencial, presentándose debilidades en esta variable. Por otra parte, se observo la posición de los docentes en la cual expresaron casi nunca el directivo los vinculan con los elementos de la responsabilidad social. Luego de se recomienda implementar los lineamientos para la Gestión Gerencial del director y la Responsabilidad Social en las organizaciones educativas; ya que los directivos requieren implementar acciones que conduzcan a la formación de equipos de trabajos para planificar, ejecutar y evaluar actividades en beneficio del colectivo

\section{Referencias}

Collertte Pirre y Delisle Gilles. (2001). La planificación del cambio. Estrategias de adaptación para las organizaciones. México: Trillas.

Chávez, N. (2006). Introducción a la investigación educativa. Caracas: Editorial Ars Grafica.

Chiavenato, I. (2006). Administración de los recursos humanos. México: Editorial Thompson.

Fernández, R. (2001). Manual para elaborar un plan de mercadotecnia. Un enfoque Latinoamericano. México: Internacional Thompson Editores.

Finol, G. (2001). Elaboración de proyectos. Caracas: Ediciones 2000- 2001.

García, S. y Dólar, S. (2003). La dirección por valores para animar la empresa en entornos turbulentos. 5ta Edición. México: Editorial McGraw-Hill. 
Gento, P. (2000). Instituciones educativas para la calidad total. 2da Edición. México: Editorial Muralla.

Hernández, R. Fernández, C. Baptista, P. (2006). Metodología de la investigación. México: McGraw-Hill.

Hurtado, J. (2000). Metodología de la investigación holística. Caracas: Sypal.

Mosley, D. Megginson, L. y Pietri, P. (2005). La supervisión. México: Editorial Thompson.

Münch y Galindo (2003). Fundamentos administrativos. (Decimotercera reimpresión). México: Editorial Trillas.

Libro verde de la Comisión Europea. (2005). Fomentar un marco europeo para la responsabilidad social de las empresas. [Documento en Línea]. [Disponible: itemsweb.esade.edu/wi/research/iis/pdfs_web/Libro_Verde.pd] [Consulta: 2013, enero, 16].

Pérez, G. (2000). Investigación cualitativa. Retos e interrogantes. Barcelona: La Muralla S.A.
Kerlinger, E (2002). Investigación del comportamiento. México: McGraw-Hill.

Pérez, S. Lombardo (1997). Guía práctica auto instruccional para la elaboración de proyectos de investigación. Delcupa, C.A. Caracas: San Bernardino.

Koontz, H.; Weihrich, H. (2006). Administración. Una perspectiva global. México: Editorial McGraw-Hill.

Requeijo, D. y Lugo, A. (2013). Administración escolar. Editorial Biosfera

Robbins, S. (2004). Comportamiento organizacional. México: Editorial Prentice Hall.

Ruetter, I. y Conde, M. (2004). Supervisión educativa. México: Editorial Fedupel.

Sierra Bravo, R. (1991 b.). Técnicas de investigación social. (7ª ed.). Madrid: Paraninfo. 